\title{
SUMMARY REPORT FOR THE FY-2015 SACSESS COLLABORATION
}

Dean R. Peterman

Bruce J. Mincher

September 2015

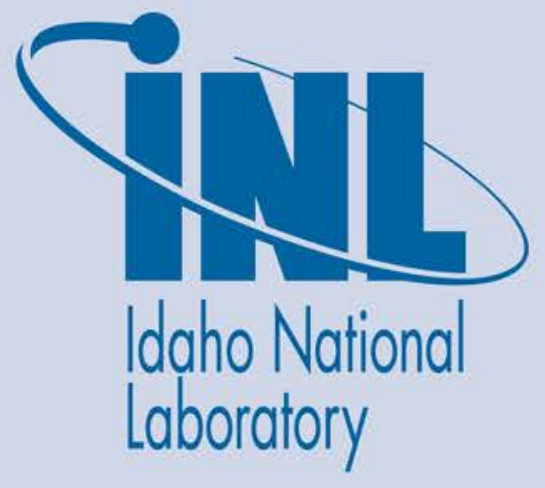

The INL is a U.S. Department of Energy National Laboratory operated by Battelle Energy Alliance 
INL/EXT-15-36606

\title{
SUMMARY REPORT FOR THE FY-2015 SACSESS COLLABORATION
}

\author{
Dean R. Peterman and Bruce J. Mincher
}

September 2015

Idaho National Laboratory
Idaho Falls, Idaho 83415

http://www.inl.gov

Prepared for the

U.S. Department of Energy

Office of Nuclear Energy

Under DOE Idaho Operations Office

Contract DE-AC07-05ID14517 


\section{INTRODUCTION}

During FY-2015, a collaborative research program was established by the Department of EnergyNuclear Energy (DOE-NE) Material Recovery and Waste Form Development program and the European Union (EU) Safety of Actinide Separation Processes (SACSESS) program. One component of this collaboration was the evaluation of the radiolytic stability of a Selective ActiNide Extraction (SANEX) separation which utilized a TODGA-based organic solvent and an aqueous phase containing the hydrophilic complexing reagent, $\mathrm{SO}_{3}$-Ph-BTP. To best simulate process conditions, this experiment was irradiated in the radiolysis/hydrolysis test loop located at the Idaho National Laboratory.

Two test loop irradiations were performed. The first irradiation involved an organic phase comprised of $0.2 \mathrm{M} \mathrm{N,N,N}, N^{\prime}$-'tetraoctyl diglycolamide (TODGA) $/ 5 \%$ octanol/dodecane in contact with a $4.5 \mathrm{M}$ $\mathrm{HNO}_{3}$ aqueous phase. The second irradiation utilized the same organic phase composition, but the aqueous phase was replaced with a solution containing $0.018 \mathrm{M} \mathrm{SO}_{3}$-Ph-BTP dissolved in $0.35 \mathrm{M} \mathrm{HNO}_{3}$. For both irradiation experiments, stable strontium, ruthenium, and neodymium were added to the aqueous phases.

Over the course of each experiment, samples of the aqueous and organic phases were removed from the test loop for further analyses. The analyses to be performed at the INL included phase disengagement time, concentration of stable metals in the aqueous and organic phases, and the determination of the distribution ratios of americium, cerium and, europium as a function of absorbed dose. The variation of the solvent composition and the concentration of the aqueous BTP as a function of absorbed dose will be determined by the EU collaborators.

\section{EXPERIMENTAL}

The irradiation source (see Figure 1) is a MDS Nordion GammaCell 220 Excel selfcontained ${ }^{60} \mathrm{Co}$ gamma irradiator. The centerline gamma dose rate in the sample chamber is $\sim 4.5 \mathrm{kGy} / \mathrm{hr}$. The solvent irradiation loop is based upon a coil of borosilicate glass tubing (0.375" OD, 0.202" ID) which is placed in the gamma irradiator sample chamber. The effective absorbed gamma dose rate of 3.3 $\mathrm{kGy} / \mathrm{hr}$ delivered to samples in the test loop was based upon decay-corrected Fricke dosimetry, ${ }^{[1]}$ the photo-bleaching of a methyl red solution due to gamma irradiation, ${ }^{[2]}$ and the duration of each irradiation. During the solvent irradiation, the aqueous and organic phases are mixed using a centrifugal contactor

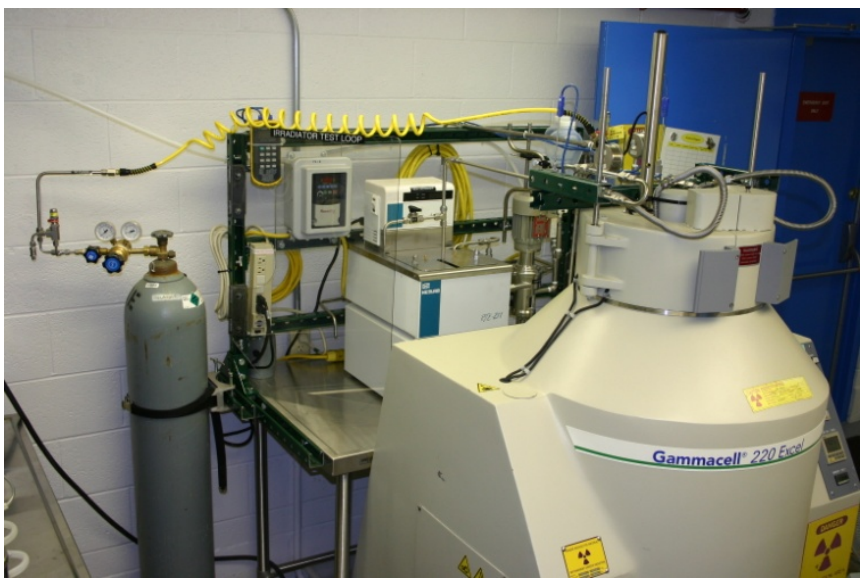

Figure 1. MDS Nordion GammaCell 220 gamma irradiator. (CINC V-02, USA) with the rotor replaced by a four vane mixing paddle. The organic and aqueous phases were metered into the mixing region of the contactor at an aqueous to organic phase volume ratio of $\mathrm{O} / \mathrm{A}=1$. The mixed phases are pumped through the irradiator test loop by a magnetic drive gear pump. The mixed phases flow through the glass coil in the irradiator sample chamber, through an external coil in a water bath, and return to the inlet of the centrifugal contactor where the phases are mixed and circulated back through the loop. In-line tube 
mixers (TAH Industries, Inc.) are used to provide additional phase mixing in the test loop. The in-line tube mixers are placed between the outlet of the gear drive pump and the inlet of the irradiation loop and between the outlet of the irradiation loop and the inlet to the external temperature control coil. The flowrate of the dispersion is in the range of $1.5 \mathrm{~L} / \mathrm{min}$ in order to maintain turbulent flow and to keep the phases dispersed. The radiolysis/hydrolysis test loop is equipped with pressure gauges and thermocouples before and after the gamma irradiator. Two flow sight glasses are used to monitor the extent of mixing in the test loop. The two phases continue to circulate until the desired dose is obtained.

A schematic view of the test loop is shown in Figure 2.

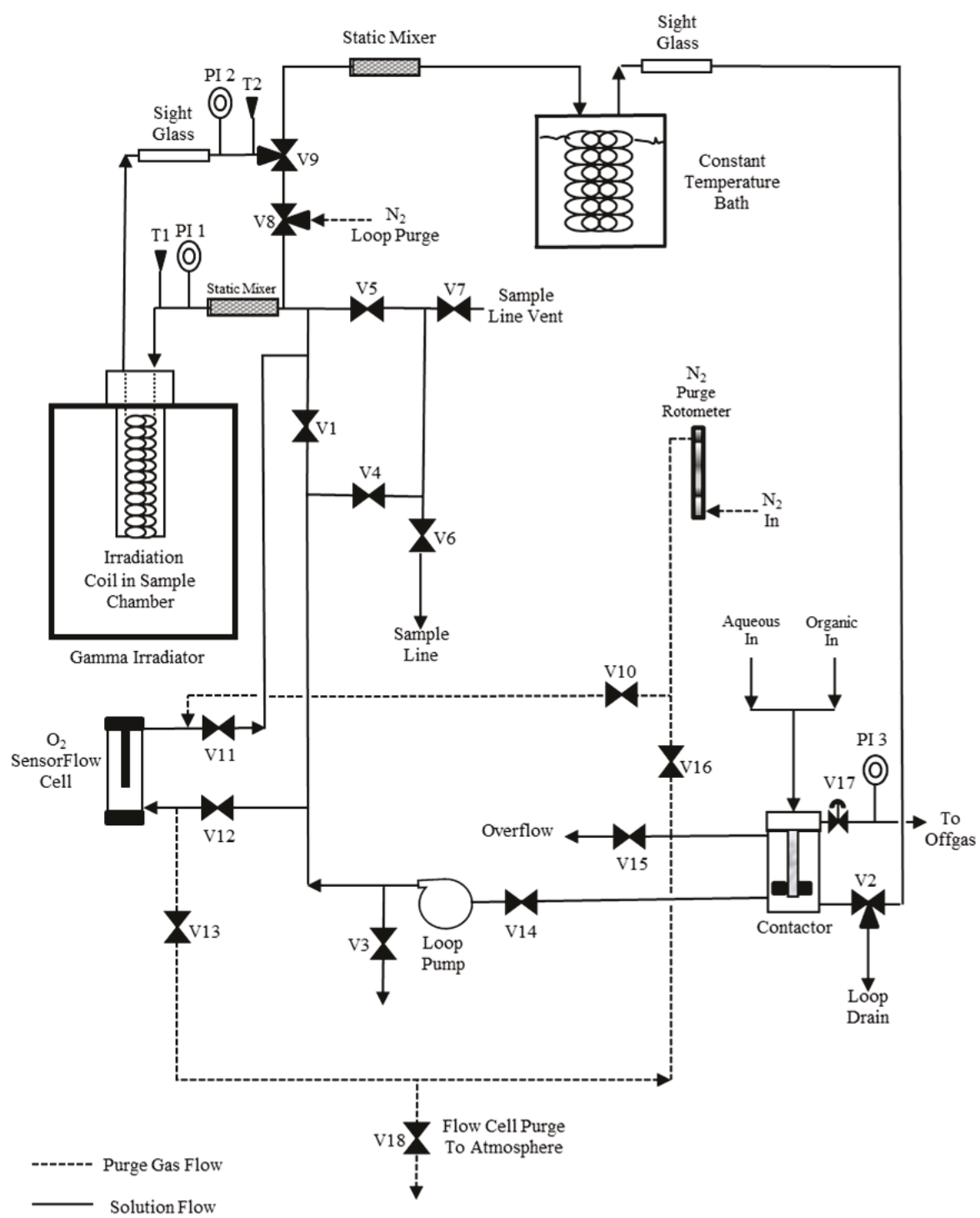

Figure 2. Schematic diagram of the INL Radiolysis and Hydrolysis Test Loop.

Preliminary samples were also irradiated in a static configuration. For static irradiation experiments, samples (aqueous, organic, or aqueous and organic) were held in septa-sealed scintillation vials and exposed to gamma irradiation using the GammaCell 220E irradiator which is equipped with a jig to hold the vials in fixed positions. The samples were sparged with air at a flow rate of $1 \mathrm{sccm}$ using mass flow 
controllers (Sierra Instruments). The gamma dose rate delivered to each position on the irradiation jig was determined by standard Fricke dosimetry. ${ }^{[1]}$

In order to gauge the effect of gamma radiolysis upon the extraction performance of the TODGA based solvents used in this study, americium, cerium, and europium distribution ratios were measured as a function of absorbed dose. Radiotracers $\left({ }^{241} \mathrm{Am},{ }^{139} \mathrm{Ce}\right.$, and $\left.{ }^{152,154} \mathrm{Eu}\right)$ were obtained from laboratory stocks. Samples of the aqueous and organic phases were obtained and radiotracers were added to the aqueous phase. Following the addition of the radiotracers, the phases were mixed for thirty minutes using a large capacity mixer (Glass-Col) and centrifuged to separate. The concentration of radiotracers present in the organic and aqueous phases was determined by gamma spectrometry. An organic to aqueous phase volume ratio of $\mathrm{O} / \mathrm{A}=1$ was used for all contacts. The solvent extraction experiments were performed at ambient temperature $\left(21 \pm 2{ }^{\circ} \mathrm{C}\right)$. The distribution of the americium, cerium, and the europium radiotracers was determined by calculating the distribution ratio, $\mathrm{D}=[\mathrm{M}]_{\mathrm{org}} /[\mathrm{M}]_{\mathrm{aq}}$. All distribution ratio determinations were performed in triplicate.

\section{RESULTS and DISCUSSION}

The variation of the measured americium, cerium, and europium distribution ratios as a function of absorbed dose up to a maximum of $650 \mathrm{kGy}$ for the static irradiation of the TODGA solvent in contact with 4.5 $\mathrm{M} \mathrm{HNO}_{3}$ is shown in Figure 3. In all cases, the radiotracers are strongly extracted into the organic phase, over the entire absorbed dose range. Due to the unavoidably large uncertainty associated with the measurement of large values of a distribution ratio (on the order of ten percent), error bars are not presented in Figure 3. The data presented in Figure 3 demonstrates that gamma irradiation of the TODGA solvent system under static irradiation conditions in the presence of the aqueous acidic phase does not result in any statistically significant variation in the measured distribution ratios within this level of uncertainty.

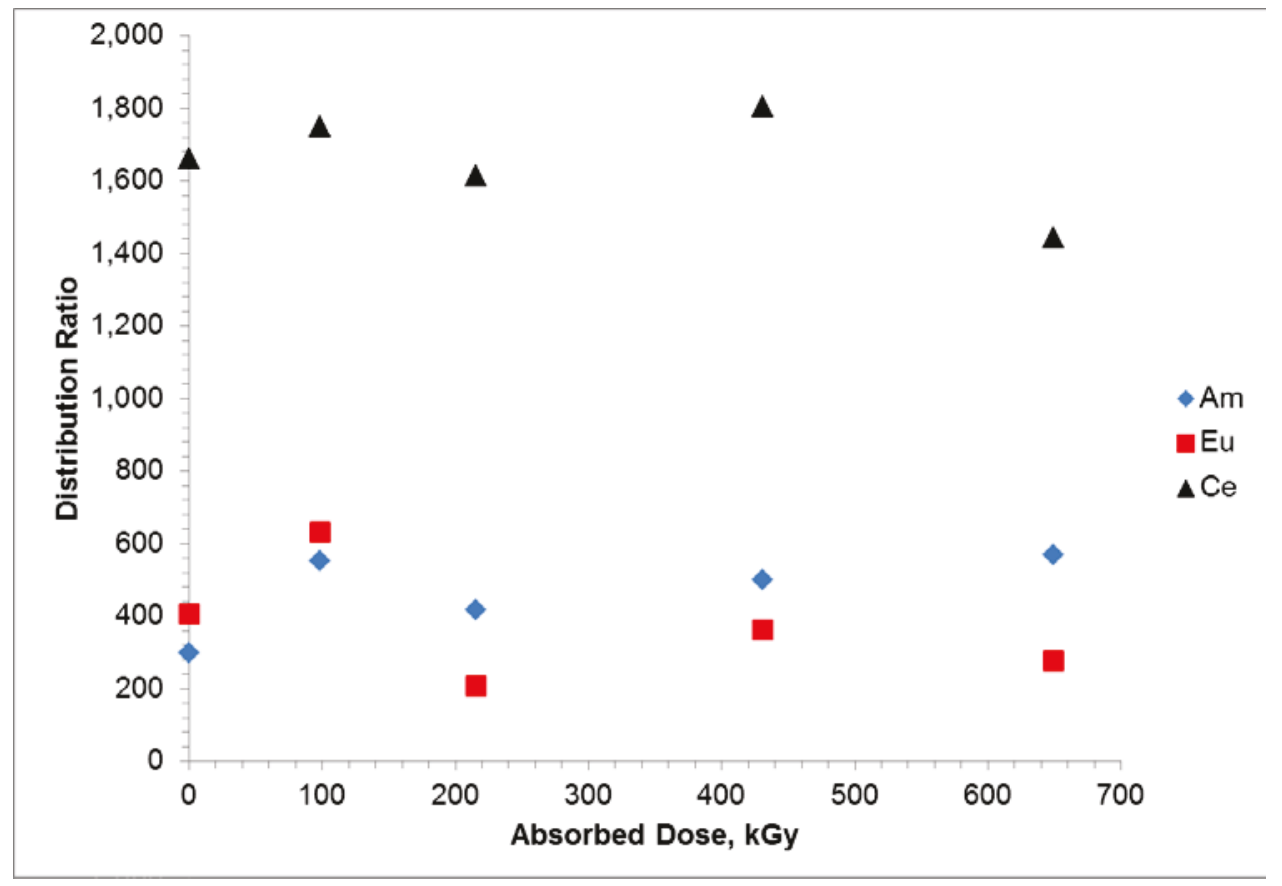

Figure 3. Distribution ratios of americium (diamonds), europium (squares), and cerium (triangles) determined using static irradiated 0.2 M TODGA / $5 \%$ octanol in n-dodecane in contact with 4.5 M $\mathrm{HNO}_{3}$ as a function of absorbed dose. 
Following the preliminary static irradiation of TODGA solvent in contact with the acidic aqueous phase, the TODGA solvent was irradiated in contact with the acidic aqueous phase in the INL test loop. The solvent was irradiated to a total absorbed dose of $878 \mathrm{kGy}$. The analyses of the samples generated during this irradiation have not been completed as of this writing. At this time, the distribution ratios of americium, europium and cerium have been determined for selected samples from early and late in the irradiation, representing low and high absorbed doses (see Figure 4). As was noted for the static irradiation experiment, no statistically significant variation in the measured distribution ratios as a function of absorbed dose occurred.

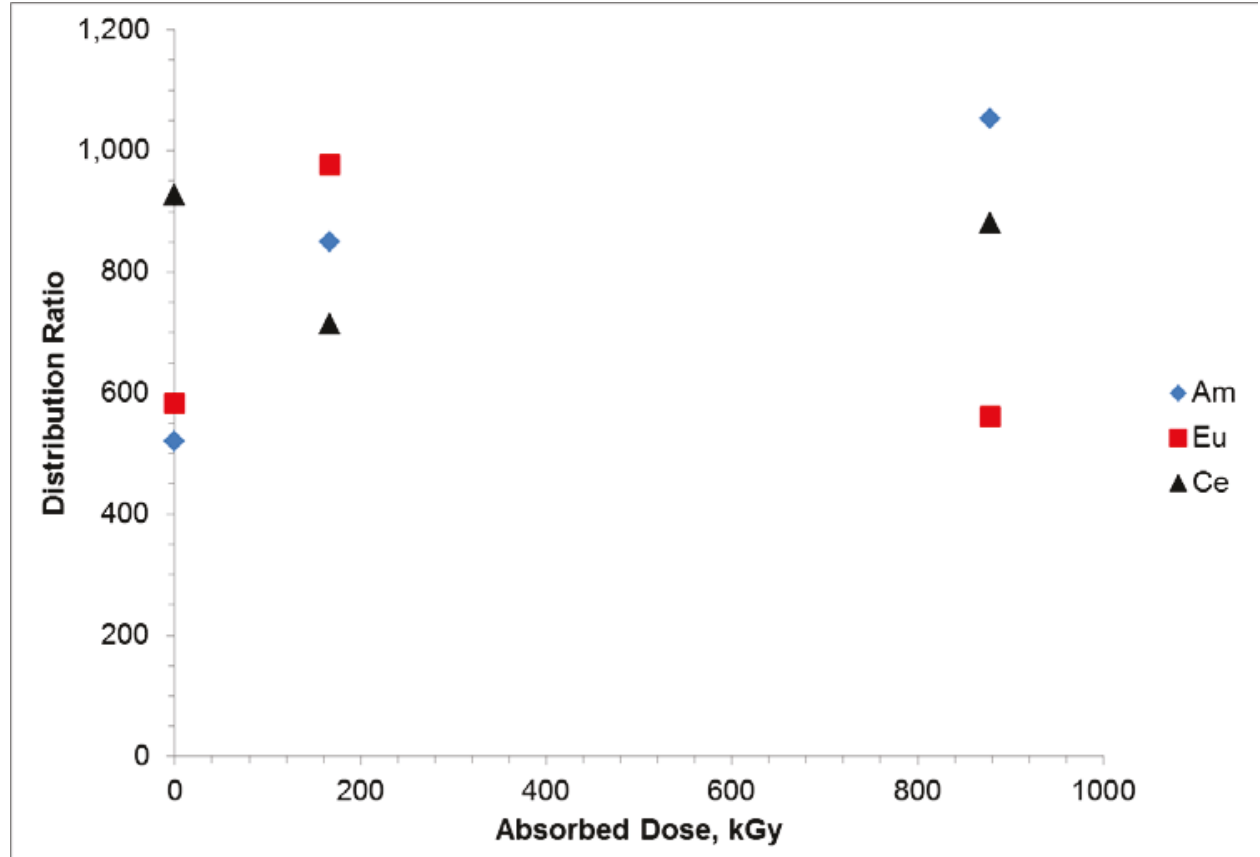

Figure 4. Distribution ratios of americium (diamonds), europium (squares), and cerium (triangles) determined using test loop irradiated 0.2 M TODGA / $5 \%$ octanol in n-dodecane in contact with $4.5 \mathrm{M}$ HNO3 as a function of absorbed dose.

The time required for the mixed phases to separate was measured at each time the test loop was sampled. Values of the dispersion number ${ }^{[3]}$ (a dimensionless number used characterize how quickly two immiscible phases separate after mixing) for the irradiated solvent have not been measured. The time required for the phases to separate increased from approximately $80 \mathrm{sec}$ at zero absorbed dose to $\sim 140 \mathrm{sec}$ following $200 \mathrm{kGy}$ absorbed dose and then exhibited a gradual decrease as the irradiation continued (see Figure 5.) 


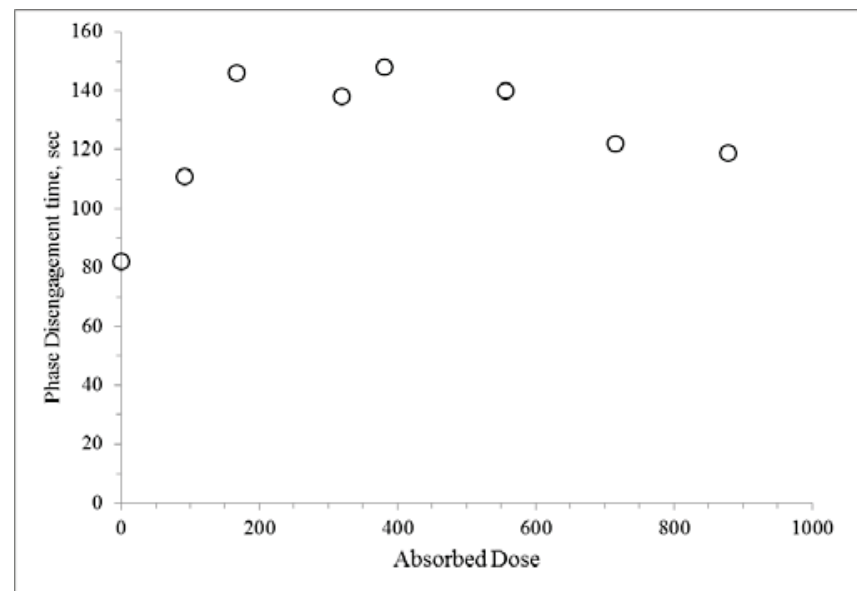

Figure 5. Plot of the time required for the 0.2 M TODGA / 5\% octanol in n-dodecane and $4.5 \mathrm{M} \mathrm{HNO}_{3}$ organic and aqueous phases to separate as a function of absorbed dose.

Prior to initiating the SACSESS test loop irradiations, the INL test loop was re-designed in order to increase the effective gamma dose rate delivered to the solvent system being tested. During the re-design, the convoluted stainless steel tubing used to connect the glass irradiation coil to the section of the test loop outside of the ${ }^{60} \mathrm{Co}$ gamma irradiator was replaced with Telfon-lined Tygon tubing. This tubing was replaced with the intention of minimizing carry-over of material from one irradiation experiment to a subsequent irradiation, which was found to be at issue in previous work. Unfortunately, this Teflon tubing underwent significant radiolytic degradation over the course of the first SACSESS test loop irradiation. The tubing was replaced during the course of the irradiation experiment, but ion chromatographic analysis of the irradiated nitric acid phase demonstrated that fluoride ion was building up in the aqueous phase as a function of increasing gamma dose (see Figure 6.) The potential exists that this accumulation of fluoride affected the distribution ratios reported in Figure 4, however, comparison with the static irradiation results in in Figure 3 indicates that any such effect must be small. Further, the distribution ratios reported in Figure 4 do not trend to lower values with increasing fluoride concentration. Following the completion of the TODGA $/ \mathrm{HNO}_{3}$ loop irradiation experiment, the Tygon tubing was replaced with stainless steel convoluted tubing. Future concerns about cross-contamination of the irradiation experiments will be addressed by the replacing the steel tubing after each experiment.

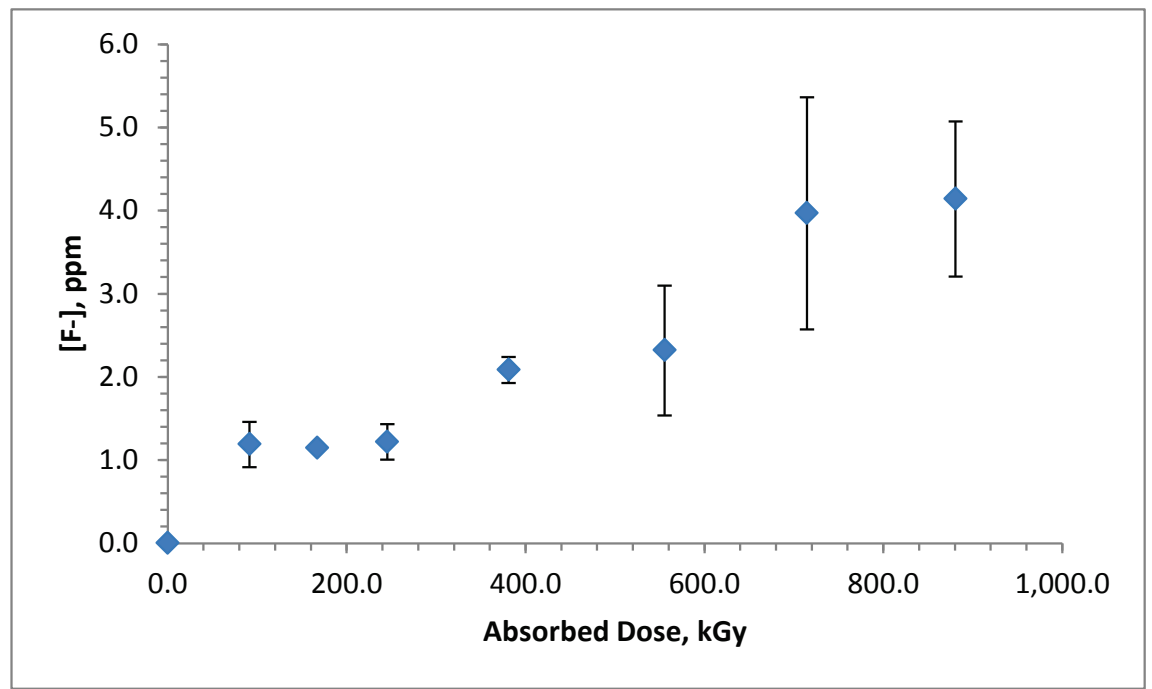

Figure 6. Concentration of fluoride ion present in the 4.5 $\mathrm{M} \mathrm{HNO}_{3}$ used for the SACSESS TODGA/ $\mathrm{HNO}_{3}$ irradiation as a function of absorbed dose. 
Am (see Figure 7). Since significant radiolytic degradation was expected, distribution ratios were determined only for three low absorbed dose and one high absorbed dose samples.

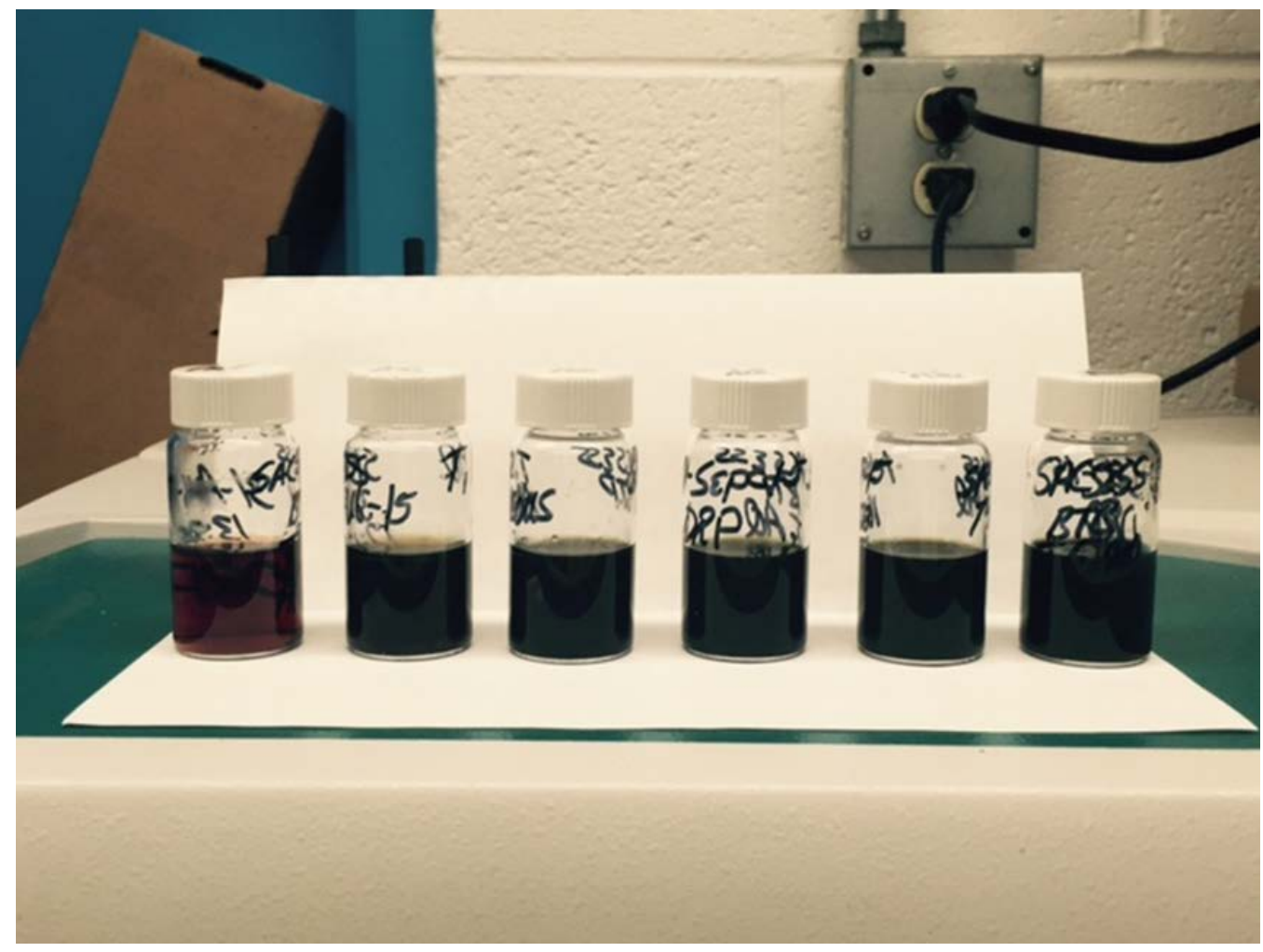

Figure 7. Aqueous samples from $\mathrm{SO}_{3}$-Ph-BTP SACSESS test loop irradiation. T0 (left) - T5 (right) Absorbed dose: T0 - 0 kGy; T1- 12.2 kGy; T2 - 25.8 kGy; T3 - 64.6 kGy; T4 - 80.8 kGy; T5 - 96.6 kGy.

The measured americium (see Figure 8) and lanthanide (see Figure 9) distribution ratios for the irradiated $\mathrm{SO}_{3}$-Ph-BTP solvent system do not exhibit a dependence upon absorbed dose that is consistent with significant radiolytic degradation of the $\mathrm{SO}_{3}-\mathrm{Ph}$-BTP ligand. At zero absorbed dose $\mathrm{D}_{\mathrm{Am}}=0.045$, but $\mathrm{D}_{\mathrm{Am}}$ increases to only 0.063 at $174 \mathrm{kGy}$ absorbed dose. Similarly, there was only a minor increase in the extraction efficiency of europium and cerium. Thus, in spite of the dramatic visual effect on the irradiated solution the separation factors between the actinide and these two lanthanides were essentially unchanged over this absorbed dose range. Separation factors $\left({ }^{\mathrm{Ce}} \mathrm{S}_{\mathrm{Am}}\right.$ and $\left.{ }^{\mathrm{Eu}} \mathrm{S}_{\mathrm{Am}}\right)$ calculated from the measured distribution ratios are presented in Table 1. 


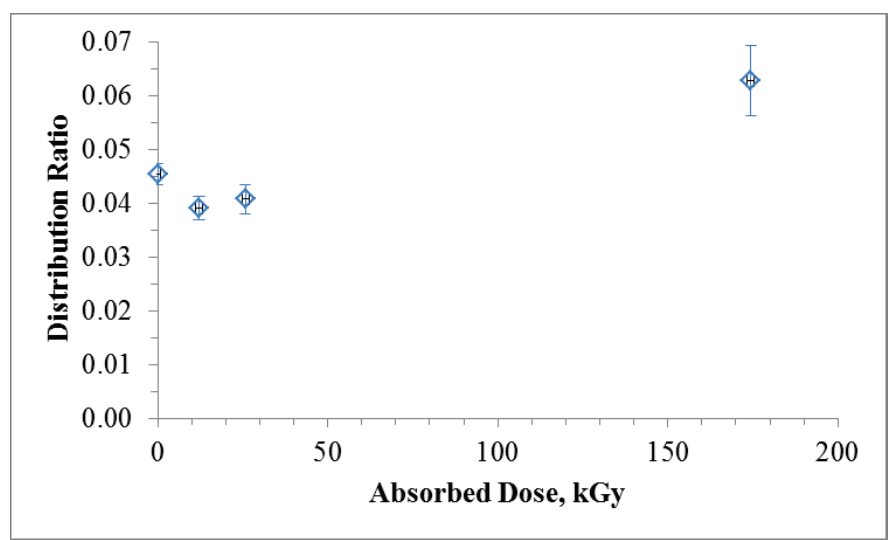

Figure 8. Values of $\mathrm{D}_{\mathrm{Am}}$ versus absorbed dose for the $\mathrm{SO}_{3}$-Ph-BTP / TODGA solvent system irradiated in the test loop.

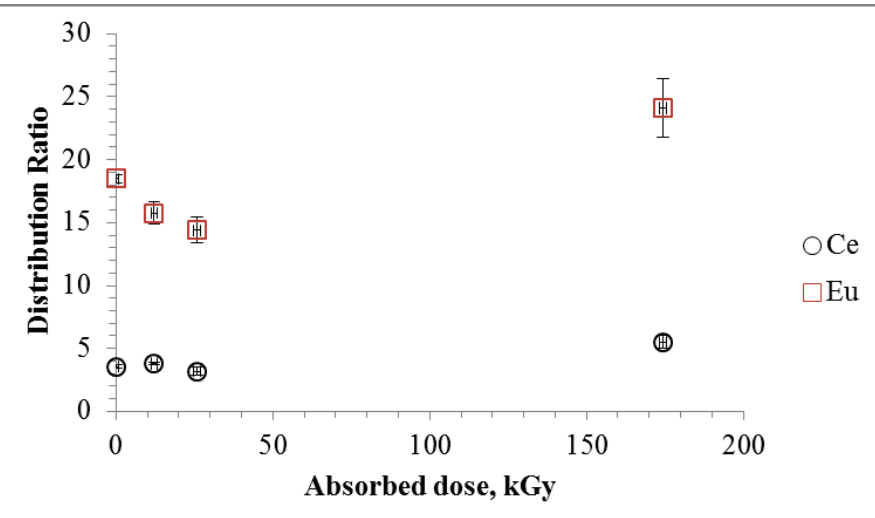

Figure 9. Values of $\mathrm{D}_{\mathrm{Ce}}$ (circles) and $\mathrm{D}_{\mathrm{Eu}}$ (squares) for the $\mathrm{SO}_{3}$-Ph-BTP / TODGA solvent system irradiated in the test loop.

Table 1. Values of the lanthanide/actinide separation factors $\left({ }^{\mathrm{Ce}} \mathrm{S}_{\mathrm{Am}}\right.$ and $\left.{ }^{\mathrm{Eu}} \mathrm{S}_{\mathrm{Am}}\right)$ for the $\mathrm{SO}_{3}-\mathrm{Ph}-\mathrm{BTP} /$ TODGA solvent system irradiated in the test loop.

\begin{tabular}{|c|c|c|c|}
\hline Sample & Absorbed Dose, $k G y$ & ${ }^{\mathrm{Ce}} \mathrm{S}_{\mathrm{Am}}$ & ${ }^{\mathrm{Eu}} \mathrm{S}_{\mathrm{Am}}$ \\
\hline $\mathrm{T} 0$ & 0 & 78.5 & 408 \\
\hline $\mathrm{T} 1$ & 12.2 & 98.0 & 404 \\
\hline $\mathrm{T} 2$ & 25.8 & 78.4 & 354 \\
\hline $\mathrm{T} 7$ & 174 & 88.1 & 384 \\
\hline
\end{tabular}

The time required for the mixed TODGA/BTP phases to separate was measured at each time the test loop was sampled. Dispersion numbers for the irradiated solvent have not been measured. As can be seen in Figure 11, the trend in the presence of the BTP-containing aqueous phase is not similar to the irradiated TODGA $/ \mathrm{HNO}_{3}$ phases. The phase separation time increased immediately upon irradiation from $35 \mathrm{sec}$ to nearly $90 \mathrm{sec}$, but then began a gradual trend to faster separation times with absorbed dose. 


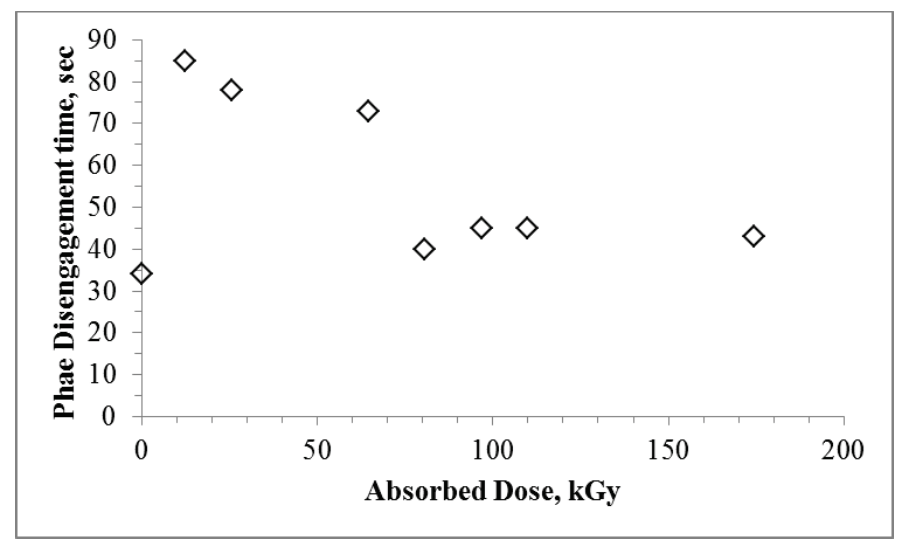

Figure 10. Plot of the time required for the $0.2 \mathrm{M}$ TODGA / 5\% octanol in n-dodecane and $0.018 \mathrm{M} \mathrm{SO}_{3}$ $\mathrm{Ph}-\mathrm{BTP}$ in $0.35 \mathrm{M} \mathrm{HNO}_{3}$ organic and aqueous phases to separate as a function of absorbed dose.

\section{CONCLUSIONS}

The effect of irradiation on a SACSESS program iSANEX formulation containing a TODGA-based organic phase and a BTP-based aqueous phase was investigated using irradiations at INL in static and test loop modes. When irradiated in contact with only the acidic aqueous phase, the TODGA organic solution maintained excellent extraction performance of americium, cerium and europium to a maximum absorbed dose of nearly 0.9 MGy. When the aqueous phase was changed to that containing the aqueous soluble BTP, the irradiated aqueous phase showed a dramatic color change, but this does not appear to have adverse effects on solvent extraction performance. Only minor increases in distribution ratios for both the lanthanides and actinide were measured, and the separation factors were essentially unchanged to a maximum absorbed dose of $174 \mathrm{kGy}$. The determination of the americium, cerium, and europium distribution ratios for the remaining SACSESS test loop samples will be completed in the near future. The analysis of stable metals concentration in the the irradiated aqueous and organic phases will be completed shortly.

\section{REFERENCES}

1. Sehested, K., The Fricke Dosimeter. In Manual on Radiation Dosimetry; Holm, N.W., Berry, R.J., Eds. Marcel Dekker, Inc: New York, 1970; pp 313 - 317.

2. Ajji, Z., Usability of aqueous solutions of mehtyl red as high-dose dosimeter for gamma radiation. Radiation Measurements 2006, 41, 438 - 442.

3. Leonard, R.A., Solvent Characterization Using the Dispersion Number. Separation Science and Technology 1995, 30, (7), 1103 - 1122. 